\title{
¿Cómo incide la actividad física en la satisfacción con la vida de los pacientes renales sometidos a hemodiálisis?
}

\author{
Rocío Polo Muñoz, Silvia Meseguer Hernández, M $^{\mathrm{a}}$ Luisa Pérez Lapuente, $\mathbf{M}^{\mathrm{a}}$ del Rosario Molina Soriano, \\ Marta López Cárceles, Juan Antonio Moreno Murcia
}

\section{Hospital Universitario Virgen de la Arrixaca. El Palmar. Murcia}

\section{Introducción:}

La enfermedad crónica contribuye a disminuir el nivel de actividad del paciente y reducir su calidad de vida. El objetivo de este estudio ha sido comprobar la relación que la tasa de actividad física, la calidad de vida y los mediadores motivacionales tenían en la predicción de la satisfacción con la vida en pacientes sometidos a hemodiálisis.

\section{Material y métodos:}

La muestra estuvo compuesta por 175 pacientes sometidos a hemodiálisis. Los criterios de inclusión fueron estar al menos seis meses en tratamiento de hemodiálisis y encontrarse en una situación clínica estable. Los instrumentos de medida utilizados han sido el Cuestionario de Calidad de Vida específico para enfermos renales que están en diálisis (KDQOL-SF), el Cuestionario de Actividad Física Habitual de Baecke, Burema, y Frijters (1982), la escala de las necesidades psicológicas básicas en general (BPNS) y la escala de satisfacción con la vida (ESDV-5). Se calcularon los estadísticos descriptivos y correlaciones de las variables del estudio. Se realizaron tres análisis de regresión lineal múltiple por pasos con el fin de analizar la predicción de la satisfacción con la vida. Los análisis se desarrollaron a través del paquete estadístico SPSS 20.0.

\section{Resultados:}

La satisfacción con la vida fue predicha en un 33\% por los mediadores psicológicos, la tasa de actividad física y la calidad de vida. Siendo la tasa de actividad física la variable que mayor poder de predicción presentó.

\section{Discusión:}

Estudios anteriores han analizado como la enfermedad renal crónica contribuye a disminuir el nivel de actividad física y la calidad de vida, y como el ejercicio físico mejora la calidad de vida. Sin embargo, no hay estudios que relacionen este conjunto de variables con la satisfacción de las necesidades psicológicas básicas y con el nivel de satisfacción con la vida. El análisis de correlaciones reveló que todas las variables se relacionaban de forma positiva con la satisfacción con la vida. Y el análisis de regresión lineal múltiple por pasos mostró que en la medida que los mediadores psicológicos básicos, la tasa de actividad física y la calidad de vida sean mayores en estos pacientes, la satisfacción con la vida será mayor. Estos resultados indican que cada uno de estos factores juega un papel importante y contribuye a favorecer la satisfacción del paciente renal con su vida en general. Muchos estudios refieren los beneficios del ejercicio a nivel fisiológico, funcional o psicológico. Aunque, en España, todavía hay escasez de investigaciones que implementen programas de ejercicio físico a pacientes en HD. Este estudio supone un primer paso para elaborar en el futuro diseños de intervención para conseguir mejorar la satisfacción con la vida a través de programas de actividad física, ya que la tasa de actividad física se muestra como el principal predictor de la satisfacción con la vida. Este trabajo resulta novedoso por la información que aporta sobre las razones de estar satisfecho con la vida. Y por la información sobre las relaciones de las necesidades psicológicas básicas con la tasa de actividad física y con la calidad de vida. 


\section{Referencias Bibliográficas}

1. Johnston, M. M., y Finney, S. J. (2010). Measuring basic needs satisfaction: Evaluating previous research and conducting new psychometric evaluations of the Basic Needs Satisfaction in General Scale. Contemporary Educational Psychology, 35, 280-296.

2. Peña Amaro, M. P. (2004). Competencia motriz del paciente urémico en programa de hemodiálisis (Tesis inédita de doctorado). Universidad de Jaén, Jaén. Segura-Ortí, E. (2010). Ejercicio en pacientes en hemodiálisis: revisión sistemática de la literatura. Nefrología, 30(2), 236-246.

3. Segura-Ortí, E., Momblanch, T., Martínez, J. F., Martí-i-Monrós, A., Tormo, G., y Lisón-Párraga, J. F. (2007). Programa de ejercicio físico para pacientes con insuficiencia renal crónica en hemodiálisis. Estudio piloto. Revista de la Sociedad Española de Enfermería Nefrológica, 10(3), 244-246.
4. Segura-Ortí, E., Rodilla-Alama, V., y Lisón-Párraga, J. F. (2008). Fisioterapia durante la hemodiálisis: resultados de un programa de fuerza-resistencia. Nefrología, 28(1), 67-72.

5. Wellard, S. (2003). Medición de la actividad física de los pacientes en tratamiento de diálisis. Método de validación. Journal of Renal Care EDTNA/ ERCA, 29(3), 143-146.

6. Zagalaz, M. L., Peña, P., Martínez, A., Mateos, C., y Martínez, M. J. (2010). The effects of physical exercise in chronic end-stage kidney failure patients on haemodialysis. Journal of Human Sport \& Exercise, 5(1), 101-113.

7. Zamojska, S., Szklarek, M., Niewodniczy, M., y Nowicki, M. (2006). Correlates of habitual physical activity in chronic haemodialysis patients. Nephrology Dialysis Transplantation, 21, 13231327. 